\title{
Methodical approach to assessing the optimal development scale of distributed cogeneration in the UPS of Russia for the long-term
}

\author{
Tatiana Pankrushina ${ }^{1 *}$, and Andrey Khorshev ${ }^{1}$ \\ ${ }^{1}$ The Energy Research Institute of the Russian Academy of Sciences, Nagornaya ul., 31-2, Moscow, Russian Federation
}

\begin{abstract}
The original methodological approach to correct assessment of the prospects and the development scale of distributed cogeneration in the long-term forecasts of the electric power industry is proposed in the article. Required suggestions for modification of the modeling tools used for the LCOEbased screening analysis and capacity expansion planning are presented. The results of application of the proposed methodological approach, which show the significant potential for distributed cogeneration development in the UPS of Russia for different tariff groups of consumers, are given.
\end{abstract}

\section{Introduction}

Recently, distributed energy has become more and more popular, and includes not only electricity generation, but also demand response technologies and energy storage technologies. It strengthens its positions both in decentralized energy supply zones and in the centralized energy systems.

Despite the fact that approaches to the definition of the distributed generation (DG) differ in the world and depend on the installed capacity of the plant and the connection voltage level, in the article DG refers to power plants with an installed capacity up to $25 \mathrm{MW}$, connected to a network at $110 \mathrm{kV}$ or less and providing the energy needs of their owners (self-consumption) or operating in the retail electricity market.

In comparison with other countries, the role of the DG in the Russian energy system is still insignificant. According to state statistics, in 2016 more than 36 thousand power plants with an installed capacity up to $25 \mathrm{MW}$ were working in Russia, and their total installed capacity reached almost $13.0 \mathrm{GW}$. Besides, over the past 10 years, the total installed capacity of such plants has increased by $30 \%$, meanwhile installed capacity of large power plants has increased only by $15.5 \%$. Thus, over 10 years, the share of DG in the total installed capacity of Russia has increased from 4.5 to $4.9 \%$, and in the production of electricity from 2.0 to $2.6 \%$.

Thermal power plants used for autonomous power supply of consumers isolated from the Unified Power System (UPS) of Russia are dominant in the structure of DG in Russia. They account for approximately $8.5 \mathrm{GW}$ (that is about $2 / 3$ of the total installed capacity of DG). The remaining $4.5 \mathrm{GW}$ are used by consumers connected to the UPS for reserving supplies from the grid and for ensuring self-consumption of electricity and heat. The most common types of DG are diesel, gas turbines, gas reciprocating engines, which are used as backup or peak, but mostly as cogeneration sources.

The geographical and climatic features of our country provide a special trend for the development of distributed generation (DG), focused more on cogeneration (DCG). Namely DCG technologies provide the most efficient use of natural gas for energy supply as well as agricultural and municipal solid waste, biomass, etc. [1].

Choice of consumers to develop their own cogeneration plants is a natural market reaction to the complexity of connection to heat and electricity networks, to high connection fees as well as to electricity and heat tariffs coupled with low quality of electricity and heat supply. With the active and mass emergence of DCG technologies (based on gas turbines, gas reciprocating engines, etc.), this trend starts to involve not only industrial, but also commercial and households consumers.

That's why under current conditions it is necessary to take into account such a rapidly growing segment in the forecasting process of the Russian electric power industry development. The ideology of regional energy systems' planning is changing and becoming more complicated to correctly match the traditional approaches of centralized and optimal planning with "flexible" solutions that have to take into account individual energy supply economic conditions of specific consumers based on DG/DCG technologies.

\section{The proposed methodical approach}

The necessity of taking into account the DG/DCG technologies at the stages of power system operation and planning requires the improvement of the methodical approach and model tools to correctly reflect the 
prospects for their development in the long-term forecasts. Slow growth of demand for electricity and heat paired with major changes in its structure by the regions and types of consumers, the rapid aging of the large power plants, as well as the strengthening requirements for cost, reliability and quality of energy supply, constant changes in pricing models on electricity and heat markets also should be taken into account.

A quantitative assessment of the potential scale of DG/DCG development is carried out in several stages, exactly the same common way as for large "system" power plants development [2]. The first stage is concerned to the competitiveness assessment of DG/DCG and alternative electricity supply options based on the levelized cost of electricity (LCOE) criterion. The second stage is devoted to estimating the potential scale of development of these technologies in the power system within a system of balances of electricity, power and heat by using the proper optimization model.

The integration of DG/DCG in this scheme is connected with a number of features:

a) technical and economic comparison of competing generating technologies moves to the end-user level. That's why it becomes essential to take into account the heat and electricity distribution costs from the different types of power plant to the final consumer. Besides, the methodical approach to correctly accounting this distribution costs in the conditions of so-called "uniform tariffs" also requires attention;

b) the efficiency of distributed generation or cogeneration for the consumer is always a commercial choice, which is determined by comparing the LCOE for its own plant and retail price [3]; thus, the final economic choice is made not through the competition of different technologies, but through competition with the retail price;

c) in this context, there is a need for a serious improvements in the model tool for the capacity expansion planning, that optimizes the system of equations representing balances of capacity, electricity and heat by the zones of UPS [2]. These improvements could give us to properly model a new DG/DCG segment of the electric power industry, that is closer to the consumers technologically and economically and participates in two retail markets at once - electricity (and power) and heat.

As already has been noted, at the first stage the areas of potential competitiveness of DG/DCG technologies are determined based on the levelized cost of electricity (LCOE) calculation. In accordance with the social efficiency approach, the LCOE indicator includes only direct material (capital and operational) costs [4].

However, for a correct assessment of the competitiveness of certain DG/DCG investment projects, implemented in the retail market, it is necessary to focus on the requirements of not only public, but also commercial efficiency. Therefore, calculation of LCOE have to take into account in addition to the direct costs the main financial and tax expenses of the investor, the real profitability and payback period of the invested capital. This methodical approach is most common in corporate planning practice, in particular in the USA [5,
6]. The general formula for calculating the LCOE for evaluating the comparative effectiveness of DG/DCG technologies could be represented as (1):

$$
L C O E=\left(F+O M+P T+\bar{K} \cdot A /(1-\operatorname{Tax})-P_{\text {heat }} \cdot Q\right) / \bar{W}
$$

where $F, O M$ - annual fuel and fixed operation and maintenance costs;

$P T$ - annual tax payments (except income tax);

Tax - income tax rate;

$P_{\text {heat }}$ - the retail heat price;

$Q$-amount of heat produced by the DCG plant;

$\bar{W}$-amount of electricity produced by the DCG plant;

$\bar{K}$ - discounted capital investments distributed over the years of construction (2):

$$
\bar{K}=\sum_{t=-T c o n s t r}^{0} K_{t} /(1+W A C C)^{t}
$$

where $K_{t}$ - capital investments by years; this value may also include additional costs for the required extension of electrical, heat and gas grids (or the connection fees);

$T_{\text {constr }}$ - construction period in years;

$W A C C$ - weighted average cost of invested capital;

$A$ - annuity coefficient which ensures equal annual payments for the return of invested capital within a given payback period $\left(T_{\text {payback }}\right)$ taking into account the value of WACC. This constant in time payment is calculated using the formula (3):

$$
A=1 / \sum_{t=1}^{T p a y b a c k} 1 /(1+W A C C)^{t}
$$

For correct comparison of alternative technologies it is essential to provide their comparability. Siting of the DG/DCG close to consumers makes it necessary to take into account, in one way or another, the cost of providing heat and electricity to the final consumer for other technologies. For example, to estimate the competitive areas of the DG/DCG in each regional power system, its LCOE is compared with the retail prices of electricity and heat. Such a comparison could be done with details, taking into account the range of retail prices for different tariff groups of consumers by voltage levels (HV - high voltage, MV1 - medium voltage 1 , MV2 - medium voltage $2, \mathrm{LV}$ - low voltage) or in aggregate - based on their weighted average value. Forecasts of retail prices' changes due to the growth of wholesale prices of electricity (including capacity) and distribution tariffs, as well as various decisions on the cross-subsidies values also have to be taking into account.

System-wide assessment of the DCG development scale was carried out by using the linear dynamic optimization model of the Russian power industry development (EPOS) [2], which was devised at the ERI RAS and now plays an important role in the SCANER integrated info-modeling complex. The emergence of the new class of generating plants requires serious improvements of the model, which is used to optimize the system of balances of power, electricity and heat:

- in the system of balance equations, there is not only the "wholesale" level, but also the "retail" level (the level of the final consumers), at which the operating conditions of the DCG power plants should be described. 
At the same time, additional components of distribution costs and costs of connection to the power system are taken into account to correctly assess the optimal development scale of the DCG;

- describing balance conditions for heat supply, it is necessary to ensure comparability of the competitive generation technologies of different types and sizes. This was done by grouping all settlements depending on the heat load mode as well as population and its density. Furthermore DCG objects as well as alternative heat sources (boilers) are explicitly presented in the model as decision variables. In this case, for large CHP plants, it is necessary to take into account the cost characteristics of heat networks (e.g. costs, losses). At the same time, for the DCG plants the consideration of such heat networks is not required.

As a result, to reflect the required features of the DCG development in the mathematical formulation of the optimization model, it is necessary to specify the condition of the annual electricity balance for the year $t$ for each regional power system $r$. This condition should be represented as the following system of equations (4) ${ }^{\mathrm{a}}$, where the first equation describes the condition of the electricity balance at the "wholesale" level, and the second one at the "retail" level.

$$
\left\{\begin{array}{l}
\sum_{i} W_{i, r, t}+\sum_{s}\left(W_{s, r, t}^{\text {exist }}+W_{s, r, t}^{\text {new }}\right)=E_{r, t}^{e l . n e t}+ \\
+\sum_{s}\left(W_{r, s, t}^{\text {exist }}+W_{r, s, t}^{\text {new }}\right), i \notin I^{D G} \\
\left(1-K_{e l(r)}^{\text {losses }}\right) \cdot E_{r, g}^{e l . n e t}+\sum_{i} W_{i, r, t}=E_{r, t}^{e l} /\left(1+K_{e l(r)}^{\text {losses }}\right), i \in I^{D G}
\end{array}\right.
$$

where

$W_{i, r, t}$ - amount of electricity produced by generation technology $i$ in the regional power system $r(\mathrm{D})$;

$W_{r, s, t}^{\text {exist }}$ - amount of electricity transmitted through the existing interconnection between regional power systems $r$ and $s$ (D);

$W_{r, s, t}^{\text {new }}$ - amount of electricity transmitted through the new interconnection between regional power systems $r$ and $s(\mathrm{D})$;

$$
K_{e l(r)}^{\text {losses }} \text { - average electricity distribution loss factor }
$$
in the regional power system $r$, which can be assumed on the basis of the previously reported data $(\mathrm{C})$;

$E_{r, t}^{\text {el.net }}$ - amount of electricity supplied from the "wholesale" to the "retail" level in the regional power system $r(\mathrm{D})$;

$E_{r, t}^{e l}$ - forecasted electricity demand (incl. exportimport surplus) in the regional power system $r(\mathrm{R})$.

The balance condition at the "wholesale" level ensures equality between:

- the electricity produced by the "large" system-scale generation $\left(i \notin I^{D G}\right)$ adjusted by the balance of electricity

\footnotetext{
a There are three types of terms in the equations: decision variables, right-hand sides, and coefficients. After the definition of these items, the type is indicated using (D) for decision variables, $(\mathrm{R})$ for right-hand sides, and $(\mathrm{C})$ for coefficients.
}

exchange with adjoining power systems;

- and the electricity supplied from the "wholesale" to the "retail" level.

The balance condition at the "retail" level guarantees electricity demand by providing it from the "wholesale" level (taking into account distribution losses) and electricity production by DG/DCG plants $\left(i \in I^{D G}\right)$.

To correctly describe the DCG features from the point of view of heat supply, it is necessary to create an annual (for year $t$ ) balance condition of consumption and production of heat by CHP of various capacities in the power system $r$ in the form of equations (5). The first equation describes the balance condition between heat supplied to the heat mains and its production by large CHPs and boilers $\left(i \notin I^{D G}\right)$. The second equation ensures that the final heat demand is satisfied by supply from the heat mains (taking into account distribution losses) and heat production by DCG $\left(i \in I^{D G}\right)$.

$$
\left\{\begin{array}{l}
\sum_{i \notin I^{D G}} q_{i, r, t} \cdot X_{i, r, t}+\sum_{i \in I_{1}^{\text {boiler }}} H_{i, r, t}^{\text {boiler }} \cdot G_{i, r, t}^{\text {new }}=E_{r, t}^{\text {heat.net }} \\
\left(1-K_{\text {heat }(r)}^{\text {losses }}\right) \cdot E_{r, t}^{\text {heat.net }}+\sum_{i \in I_{2}^{\text {boiler }}} H_{i, r, t}^{\text {boiler }} \cdot G_{i, r, t}^{\text {new }}+ \\
+\sum_{i \in I^{D G}} q_{i, r, t} \cdot X_{i, r, t}=E_{r, t}^{\text {heat }} /\left(1+K_{\text {heat }(r)}^{\text {losses }}\right)
\end{array}\right.
$$

where

$q_{i, r, t}$ - specific annual heat production per unit of installed electrical capacity of CHP technology $i(\mathrm{C})$;

$X_{i, r, t}$ - installed electrical capacity of CHP technology $i$ (D);

$K_{\text {heat }(r)}^{\text {losse }}$ - average heat distribution loss factor in the regional power system $r$, which can be assumed on the basis of the previously reported data $(\mathrm{C})$;

$E_{r, t}^{\text {heat.net }}$ - amount of heat supplied from large CHPs and boilers ( $i \notin I^{D G}$ ) to the heat mains in the regional power system $r(\mathrm{D})$;

$E_{r, t}^{\text {heat }}$ - forecasted heat demand in the regional power system $r(\mathrm{R})$;

$G_{i, r, t}^{\text {new }}$ - thermal capacity of the new boiler technology $i$ (D);

$H_{i, r, t}^{\text {boiler }}$ - thermal capacity utilization factor of the new boiler technology $i$ (hours per year) (C);

$I_{1}^{\text {boiler }}, I_{2}^{\text {boiler }}$ - sets of boiler technologies considered as alternative for large CHPs $\left(i \notin I^{D G}\right)$ and for DCG plants $\left(i \in I^{D G}\right)$, respectively.

Usually, the solution of the optimization model of the whole power industry development is obtained by minimizing the cost (objective) function, which is the total discounted costs of meeting the forecasted demand for electricity, power and heat in the UPS of Russia, taking into account the prolonged effect of investment decisions.

But the inclusion of DCG technologies in the optimization model requires to accounting in the cost function the total costs of heat transport and electricity 
distribution from "wholesale" to "retail" level for the entire forecast period. It could be done by adding the following summand (6):

$$
\begin{aligned}
& C_{\text {opt }}=\sum_{t} \sum_{r}\left(k_{r, t}^{e l . n e t} \cdot E_{r, t}^{e l . n e t}+k_{r, t}^{\text {heat.net }} \cdot E_{r, t}^{\text {heat.net }}\right) \cdot \frac{1}{(1+d)^{t}} \text { (6) } \\
& \text { where } \\
& \quad d \text {-discount rate (C); } \\
& \quad k_{r, t}^{\text {el.net }} \text { - cost (per unit) of the electricity distribution }
\end{aligned}
$$

from the "wholesale" to the "retail" level in the regional power system $r$ in year $t$. In the absence of data, their value can be estimated as a weighted average of the previously reported tariffs for electricity transmission services for certain groups of consumers by voltage levels (HV, MV1, MV2, LV) (C);

$k_{r, t}^{\text {heat.net }}$ - cost (per unit) of the heat distribution from large CHP plants and boilers in the regional power system $r$ in year $t$. In the absence of data, their values could be estimated as a weighted average of the previously reported tariffs for services for the heat distribution within the boundaries of the regional power system (C).

\section{Verification of the proposed methodical approach}

To assess the competitiveness of DG/DCG technologies, it is necessary to prepare their key cost and performance characteristics: such as capital, fixed operation and maintenance costs, efficiency (heat rate) and electricity for own consumption.

Required data were estimated on the base of the proposals of equipment suppliers for DCG commercial projects and analysis of the structure of overnight capital costs from foreign reviews $[7-10]$. They are shown in Table 1.

Table 1. The cost and performance data of distributed cogeneration technologies

\begin{tabular}{|c|c|c|c|}
\hline & Units & Gas engine & $\begin{array}{c}\text { Gas } \\
\text { turbine }\end{array}$ \\
\hline Unit capacity & MW & 1 & 1 \\
\hline $\begin{array}{c}\text { Overnight } \\
\text { capital costs }\end{array}$ & $\begin{array}{c}\text { RUB } \\
2018 / \mathrm{kW}\end{array}$ & $\begin{array}{c}81400- \\
97700\end{array}$ & $\begin{array}{c}74000- \\
88800\end{array}$ \\
\hline Fixed O\&M & $\begin{array}{c}\% \text { of capital } \\
\text { costs }\end{array}$ & 5,0 & 4,5 \\
\hline $\begin{array}{c}\text { Electrical } \\
\text { efficiency }\end{array}$ & $\%$ & $41-42$ & $30-32$ \\
\hline
\end{tabular}

The obtained ratio of capital costs of gas reciprocating engines and gas turbines are well corresponded with foreign and domestic estimates capital cost of gas engines is higher than gas turbines by about $10-14 \%$. As for DG plants (producing an electricity only), their capital costs were as $80 \%$ of the DCG plants, based on similar estimates for large-scale generation.

Areas of competitive DCG applications were estimated for $1 \mathrm{MW}$ DG and DCG plants with gas turbines and gas reciprocating engines for five regions of central Russia (Ivanovo, Kaluga, Lipetsk, Ryazan and Tambov regions). Heat prices for each of these areas were calculated as levelized costs of heat from new boiler (so called "alternative boiler" method [11]). The range of heat tariffs calculated with the "alternative boiler" method is 1,607-1,711 rubles per Gcal. Retail gas prices for DCG and boilers in each region, ranged from 5580 to 6376 rubles / tce (excl. VAT), was also used for the calculation.

LCOE values for DCG were calculated for maximum and minimum level of capital costs (are shown in Table 1) according to formula (1). LCOE values for DG plants were also calculated according to formula (1), but revenues from heat sales were excluded from the calculation. Results of the calculation as well as the retail tariffs in all of regions mentioned above by voltage levels are presented in Table $2^{\mathrm{b}}$

Table 2. Retail electricity tariffs for different voltage levels in several regions of central Russia compared with LCOE of DG/DCG, RUB 2018/kWh

\begin{tabular}{|c|c|c|c|c|c|}
\hline $\begin{array}{c}\text { Regions of central } \\
\text { Russia }\end{array}$ & Ivanovo & Kaluga & Lipetsk & Ryazan & Tambov \\
\hline HV & 3,66 & 3,95 & 2,82 & 3,41 & 4,01 \\
\hline MV1 & 4,20 & 4,70 & 4,11 & 4,37 & 4,17 \\
\hline MV2 & 5,54 & 4,98 & 4,28 & 4,57 & 4,32 \\
\hline LV (Households) & 2,77 & 2,69 & 2,16 & 3,05 & 2,40 \\
\hline LCOE DG 1 MW & $5,01-$ & $5,30-$ & $5,12-$ & $5,09-$ & $4,99-$ \\
& 5,83 & 6,06 & 5,92 & 5,90 & 5,82 \\
\hline LCOE DCG 1 & $4,31-$ & $4,51-$ & $4,39-$ & $4,37-$ & $4,29-$ \\
MW & 5,92 & 6,08 & 5,98 & 5,97 & 5,91 \\
\hline
\end{tabular}

The relative competitiveness of DG/DCG plants was estimated as a ratio between their LCOE and retail prices for different tariff groups of consumers at different voltage levels. These results for all 5 regions are presented in Fig. 1 and Fig. 2.

These results show that the development of DG power plants (with a capacity of $1 \mathrm{MW}$ ) in the existing economic conditions is not reasonable even at low capital costs. Only for consumers connected to the grid at the medium voltage level (MV2), such DG power plants could be competitive in comparison with the electricity supply from the grid. That's true for the regions with the greatest regulatory distortions of retail prices due to cross-subsidies. For the households with the current low electricity prices the supply from the network is more preferable.

However, the second product (heat) allows improving the competitiveness of DCG power plants in the retail market compared to DG, since the total costs are partially offset by revenue from the sale of heat. Thus, the development of distributed cogeneration can become efficient even for consumers connected to medium voltage networks (MV1 and MV2), but only when capital costs of DCG is low. For consumers at the high voltage level (especially in Lipetsk and Ryazan regions) the development of such small sources is not yet reasonable. In addition, due to the current cross-

\footnotetext{
${ }^{\mathrm{b}}$ Retail tariffs for consumers of different voltage levels were corrected according to inflation rate from 2017 to 2019.
} 
subsidization and low electricity prices for households they are also not interested in the development of distributed cogeneration yet.
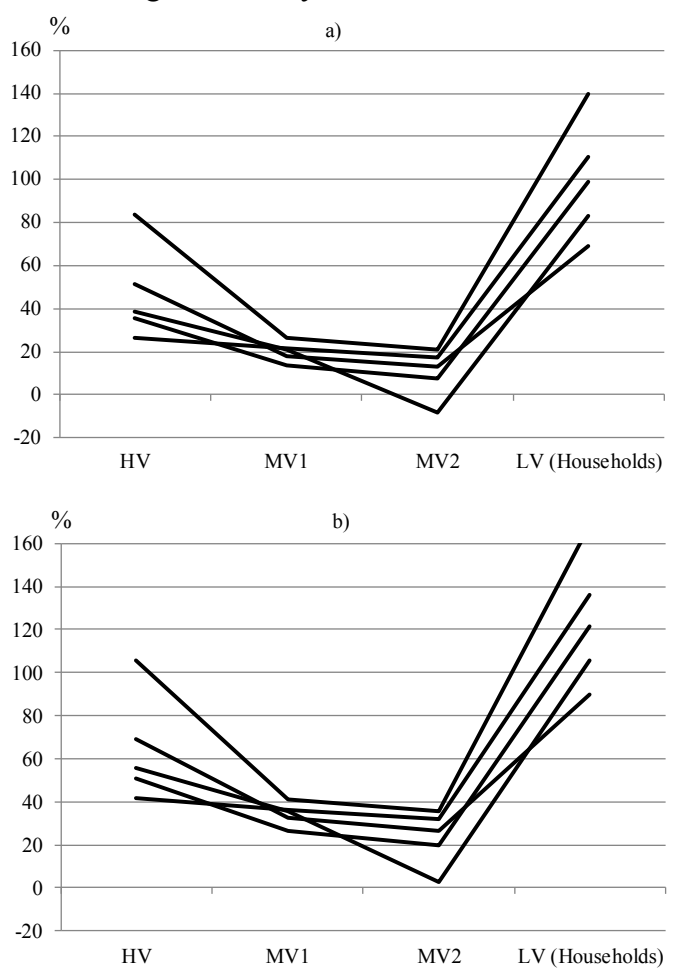

Fig. 1. The difference between LCOE of DG power plants of $1 \mathrm{MW}$ and the electricity tariffs for consumers at different voltages with minimum (a) and maximum (b) capital costs, $\%$.
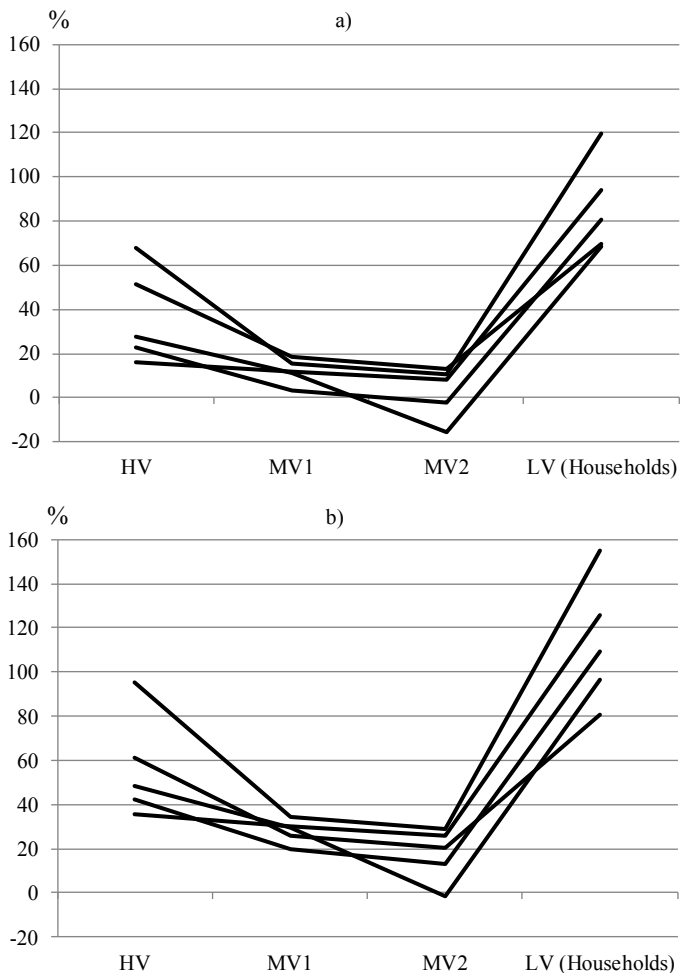

Fig. 2. The difference between LCOE of DCG power plants of $1 \mathrm{MW}$ and the electricity tariffs for consumers at different voltages with minimum (a) and maximum (b) capital costs, $\%$.

To verify the proposed methodical approach and test the changes in the capacity expansion planning optimization model (EPOS), multivariant calculations were carried out at the second stage.

Here the potential area of DCG development was limited to ensuring the increasing heat demand of cities with a population less than 100 thousand people (i.e. for energy supply of new residential areas in small cities) located in the part of the UPS of Russia within the centralized gas supply system. In accordance with the ERI RAS estimates, the potential heat demand in these areas of such cities could reach 30 million Gcal by 2030 and about 44 million Gcal by 2040. It is the heat demand that DCG plants will compete for with alternative gas boilers.

All external conditions of the Russian power industry development (demand for electricity and power, fossil fuels prices, etc.) were taken in accordance with the innovative scenario developed in 2018 at the ERI RAS. Cost and performance data of large, system-scale power plants are assumed as the minimum values of the range in Table 3, the fuel prices are presented in Table 4, and the discount rate is $7.5 \%$.

Table 3. The cost and performance data of large, system-scale, power plants

\begin{tabular}{|c|c|c|c|c|}
\hline & Units & Nuclear & CCGT & $\begin{array}{l}\text { Coal } \\
\text { SC }\end{array}$ \\
\hline Unit capacity & MW & 1255 & 400 & 660 \\
\hline \multirow{2}{*}{$\begin{array}{l}\text { Overnight capital } \\
\text { costs }\end{array}$} & \multirow{2}{*}{ RUB 2018/kW } & $\underline{106800}$ & $\underline{36000}$ & 66100 \\
\hline & & 124300 & 45000 & 73600 \\
\hline Fixed O\&M & $\%$ of capital costs & 2,2 & 3,0 & 2,9 \\
\hline \multirow{2}{*}{$\begin{array}{c}\text { Fuel costs / Heat } \\
\text { rate }\end{array}$} & RUB 2018/kWh & 0,26 & - & - \\
\hline & gce/kWh & - & 225 & 320 \\
\hline Own consumption & $\%$ & 6,5 & 3,3 & 6,9 \\
\hline
\end{tabular}

Table 4. Assumed gas and coal prices, RUB 2018/tce

\begin{tabular}{|l|c|c|c|c|c|c|c|}
\hline Region & 2018 & 2020 & 2025 & 2030 & 2035 & 2045 & 2050 \\
\hline \multicolumn{8}{|c|}{ Gas prices } \\
\hline Central & 4600 & 4780 & 5050 & 6090 & 7210 & 7870 & 8600 \\
\hline Ural & 3900 & 4020 & 4030 & 5100 & 5840 & 7370 & 6960 \\
\hline Siberia & 2900 & 3260 & 4260 & 5245 & 6040 & 6595 & 7200 \\
\hline \multicolumn{8}{|c|}{ Coal prices } \\
\hline Central & 3700 & 4100 & 3890 & 3780 & 3650 & 3640 & 3640 \\
\hline Ural & 3300 & 3600 & 3450 & 3350 & 3250 & 3250 & 3250 \\
\hline Siberia & 1100 & 1190 & 1190 & 1260 & 1270 & 1280 & 1290 \\
\hline
\end{tabular}

Based on the proposed approach and data, Fig. 3 presents the optimal scale of the DCG development in 2040.

First of all, it is important to analyze the effects of reconfiguration of the optimization model presented in part II of this article. As you can see from Fig. 3, without taking into account the cost of supplying electricity to the final consumer in the model cost function (i.e. DCG competes on the wholesale market with "large" generation), DCG development is completely ineffective, even if the its capital cost is low.

Moreover, the impact of the electricity distribution cost from the "wholesale" to the "retail" level was estimated. For each region of Russia, this cost was considered in a broad range, valued on the basis of the previously reported distribution tariffs for consumers of various groups (from $\mathrm{HV}$ to $\mathrm{LV}$ ). It allows us to estimate 
the efficiency and potential scale of DCG development among consumers of different tariff groups.

Thus, setting the electricity distribution costs even at the level of the HV tariff, makes the development of distributed cogeneration competitive in some regions. However, its development scale in 2040 will not exceed 1.4 - $2.1 \mathrm{GW}$. Obviously, with the growth of the distribution tariff (from HV to LV group), the effective scale of DCG development is going to increase. It will reach its limit (about $10 \mathrm{GW}$ ) at the LV tariff. This limit is determined by the assumed heat demand of new residential areas in small cities.

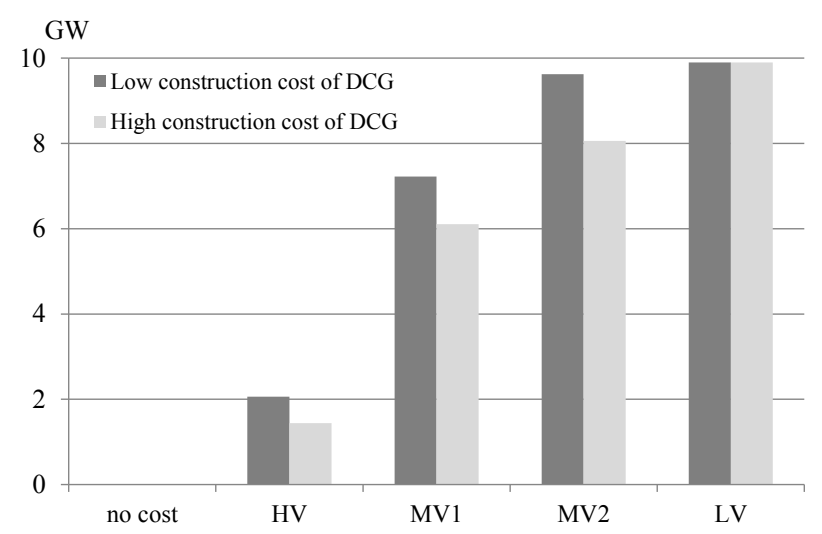

Fig. 3. The optimal development scale of DCG in UPS of Russia in 2040 depending on DCG construction cost and electricity distribution cost from "wholesale" to "retail" level, GW.

The high uncertainty of the DCG capital costs makes this factor crucial in determining the optimal scale of its development. However, as can be seen from Fig. 3, the DCG capital costs variation in the assumed range has a rather moderate impact on its optimal development scale. For example, considering the electricity distribution tariffs at the level of MV1 and MV2, the installed capacity of the DCG rises by about $20 \%$ with decrease of its capital costs.

Hence, the competitive areas of DCG development obtained during the calculation of LCOE were confirmed by the results of optimization.

\section{Conclusions}

The trend of booming distributed generation development that has been observed in recent years in many countries is beginning to reveal in Russia. Taking into account climatic and geographical conditions of the country, the most reasonable way of development is distributed cogeneration, especially based on natural gas. Thus the features of distributed cogeneration have to be taken into account in researches of the Russian electric power industry development for the long term.

The proposed methodical approach is an attempt to combine the traditional approaches of centralized and optimal planning with "flexible" solutions to consider the individual characteristics of the economic conditions of energy supply of different types of consumers.

The main distinctive features of this methodical approach is in substitution of the "decision-making level", i.e. the criteria of the effective investment decisions move from the "wholesale" (system-wide) level to the "retail" level (end-user level).

The calculations showed the rather large potential of the distributed cogeneration development for industrial, commercial and agricultural consumers connected at medium voltage (MV1, MV2).

\section{Acknowledgment}

The research was supported by the Russian Science Foundation (project No. 17-79-20354).

\section{References}

1. Distributed Energy Resources in Russia: Development Potential (Moscow School of Management SKOLKOVO, 2018). https://energy.skolkovo.ru/downloads/documents/SEneC /Research/SKOLKOVO_EneC_DER_2018.10.09_Eng.p df

2. F. Veselov, E. Volkova, A. Kurilov, A. Makarova, A. Khorshev. Bulletin of the Russian Academy of Sciences. Energy, 4, 82-94 (2010).

3. T. Pankrushina, A. Solyanik, I. Zolotova. Proc. of the International Scientific Conference "Far East Con" (ISCFEC 2018). DOI: 10.2991/iscfec-18.2019.155.

4. Projected Costs of Generating Electricity (OECD/IEA, 2015).

5. Cost Estimation Methodology for NETL Assessments of Power Plants Performance. Quality guidelines for energy system studies (DOE/NETL, 2011).

6. World Energy Perspective. Cost of Energy Technologies. 2016 (UK, WEC/BNEF, 2016).

7. Distributed Generation and Combined Heat \& Power System Characteristics and Costs in the Buildings Sector (U.S. Department of Energy, Washington, DC, 2017).

8. Catalog of CHP Technologies. Section 2. Technology Characterization - Reciprocating Internal Combustion Engines (U.S. Environmental Protection Agency, Combine Heat and Power Partnership, 2015).

9. Technology Data for Energy Plants for Electricity and District heating generation. November 2018 update (Danish Energy Agency, 2018).

10. M.J. Schmeida. Power Engineering, 122, 1 (2018).

11. Alternative boiler calculator (The Ministry of Energy of Russia, 2018). https://instrumentak.minenergo.gov.ru. 